\title{
Disparities in perinatal health: what can we do?
}

\author{
Zaneta Forson-Dare $\mathbb{C}^{1,2} \cdot$ Leslie M. Harris ${ }^{1,2} \cdot$ Patrick G. Gallagher ${ }^{1,2}$
}

Received: 4 January 2021 / Revised: 13 January 2021 / Accepted: 14 January 2021 / Published online: 28 January 2021

(c) The Author(s), under exclusive licence to Springer Nature America, Inc. 2021

The evidence for the impact of structural racism on health outcomes is overwhelming. Formulating an effective mitigating response has been difficult for healthcare workers. Last month's issue of the Journal of Perinatology focused on the broad issues of health disparities, contributing factors, and resulting in increased mortality and morbidity, underscoring this as a public health emergency. Racial disparities are compounded by gender and social disparities, affecting Black Americans the most [1]. There are many systemic factors, such as housing, education, nutrition, environmental exposures, and the justice system, that contribute to disparities in healthcare during the course of a person's lifetime. In our roles as medical providers, we may feel we have minimal control over such factors and a sense of powerlessness to face the alarming disparities in healthcare outcomes explored in the previous issue. However, clear disparities have been identified in the quality of access to healthcare, provider-patient interactions, treatment, and overall care, which all fall under our direct responsibility in the healthcare community [2]. These are the things that we can and should work to change.

Many studies have documented racist beliefs, practices, and implicit biases among individual healthcare providers that contribute to disparities in clinical care and outcomes [3]. Minority patients are often cared for in lower quality hospitals than White patients and consequently receive a lower standard of care [4]. Structural, organizational, resource, and process-related inequalities between differing hospitals are contributing factors. Yet, even when cared for in the same hospital, researchers have

Patrick G. Gallagher

patrick.gallagher@yale.edu

1 Division of Neonatal/Perinatal Medicine, Department of Pediatrics, Yale University School of Medicine, New Haven, CT, USA

2 Yale New Haven Children's Hospital, New Haven, CT, USA documented racial disparities in outcomes [5]. In the neonatal intensive care unit, there are racial and ethnic differences in the ways patients and their families are treated that arise from conscious and unconscious biases, ultimately leading to suboptimal care [6]. Individual provider biases are compounded by the structural racism of the institutions in which they work. Because it is essential to understand the links between the provider bias and structural racism [7], the articles in this issue of the Journal of Perinatology focus on neonatal-perinatal medicine providers, neonatal intensive care units, and their healthcare institutions.

In addition to racial inequities, disparities exist due to gender, ethnicity, and social class. For instance, in neonatal-perinatal medicine, there are fewer women in leadership positions, and they continue to suffer from income inequalities, as shown by Horowitz et al. [8]. The authors also noted that the under-represented minorities working in neonatology continue to suffer from lower compensation levels, further highlighting the compounding effect of being a female and belonging to an underrepresented minority [9].

Education on the existence of systemic racism and individual biases in our profession should be woven into the structure of medical training from the very beginning. After formal training is complete, it should be a component of ongoing professional development. These issues are not the focus in most medical educational institutions, as explored by Rent [10], who also highlights how one should transform their thinking by identifying "racism" as opposed "race" as the primary healthcare-related risk factor. At the individual level, the key to improving our patient care is to understand and accept the influences of our own backgrounds, beliefs, and biases on our clinical practice. Edmonds et al. and Glazer et al. studied how these influences lead to disparities in personal interactions, triage, diagnosis, referrals, treatment, and advice medical providers give to patients and their families [11, 12]. Managing these influences requires personal awareness through education, anti-bias and anti-racism 
training, as well as some institutional standardization of care to mitigate these individual variations. At the institutional level, we must examine the systemic processes that result in disparate outcomes through quality improvement. Padula et al. point out that, given the economic dominance of many healthcare systems within the communities they serve, it is also essential that these systems act to quantify and then alleviate the barriers to care [13].

So where do we go from here? As Gautham states, we have described the situation, it is time to "internalize that it is our job as health professionals" and take action against systemic racism and other inequities in our health systems [2]. The articles in this issue present data on the roles we as medical providers play but, more importantly, the interventions in education, representation and at the individual and institutional levels that can be taken to combat disparities in health outcomes. We have the tools to battle this, we need to put them to work-our patients cannot afford to wait.

\section{Compliance with ethical standards}

Conflict of interest The authors declare that they have no conflict of interest.

Publisher's note Springer Nature remains neutral with regard to jurisdictional claims in published maps and institutional affiliations.

\section{References}

1. Noonan AS, Velasco-Mondragon HE, Wagner FA. Improving the health of African Americans in the USA: an overdue opportunity for social justice. Public Health Rev. 2016;37:12.

2. Gautham KS. I can't breathe. J Perinatol. 2020.

3. Thomas EV. "Why even bother; they are not going to do it?" The structural roots of racism and discrimination in lactation care. Qual Health Res. 2018;28:1050-64.

4. Feagin J, Bennefield Z. Systemic racism and U.S. health care. Soc Sci Med. 2014;103:7-14.

5. Howell EA, Zeitlin J. Improving hospital quality to reduce disparities in severe maternal morbidity and mortality. Semin Perinatol. 2017;41:266-72.

6. Sigurdson K, Morton C, Mitchell B, Profit J. Disparities in NICU quality of care: a qualitative study of family and clinician accounts. J Perinatol. 2018;38:600-7.

7. Matthew DB. Toward a structural theory of implicit racial and ethnic bias in health care. Health Matrix. 2015;25:61-85.

8. Horowitz E, Randis TM, Samnaliev M, Savich R. Equity for women in medicine-neonatologists identify issues. J Perinatol. 2020.

9. Horowitz E, Samnaliev M, Savich R. Seeking racial and ethnic equity among neonatologists. J Perinatol. 2021. In press.

10. Rent S. If it's important, teach us: accountability in education on structural racism. J Perinatol. 2020.

11. Tucker Edmonds B. Diverse perspectives on death, disability, and quality of life: an exploratory study of racial differences in periviable decision-making. J Perinatol. 2021. In press.

12. Glazer KB, Sofaer S, Balbierz A, Wang E, Howel EA. Perinatal care experiences among racially and ethnically diverse mothers whose infants required a NICU stay. J Perinatol. 2021. In press.

13. Padula AM, Shariff-Marco S, Yang J, Jain J, Liu J, Conroy SM, et al. Multilevel social factors and NICU quality of care in California. J Perinatol. 2021. In press. 